observations. "What's really concerning is how bright all these new satellite constellations will be," says Patrick Seitzer, an astronomer at the University of Michigan in Ann Arbor.

Within the next year or so, SpaceX plans to launch an initial set of 1,584 Starlink satellites into 550-kilometre-high orbits. At a site such as Cerro Tololo, Chile, which hosts several major telescopes, six to nine of these satellites would be visible for about an hour before dark and after dawn each night, Seitzer has calculated.

Most telescopes can deal with that, says Olivier Hainaut, an astronomer at the European Southern Observatory (ESO) in Garching, Germany. Even if more companies launch megaconstellations, many astronomers might be able to continue their work, he says. Hainaut has calculated that if 27,000 satellites are launched, then ESO's Chile telescopes will lose about $0.8 \%$ of their long-exposure observing time near dusk and dawn. “Normally, we don't do long exposures during twilight," he says. "We are pretty sure it won't be a problem for us."

But an upcoming, cutting-edge telescope could be in bigger trouble. The US Large Synoptic Survey Telescope (LSST) will use an enormous camera to study dark matter, dark energy, asteroids and other astronomical phenomena. It will survey the entire visible sky at least once every three nights, starting in 2022. Because the telescope has such a wide field of view, satellites trailing across the sky could affect it substantially, says Tony Tyson, an astronomer at the University of California, Davis, and the LSST's chief scientist.

He and his colleagues have been studying how up to 50,000 new satellites - an estimate from companies' filings with the US government - could affect LSST observations. Early findings suggest that the telescope could lose significant amounts of observing time to satellite trails near dusk and dawn.

\section{Paint it black}

There are other impacts beyond losing observing time. Bright satellite streaks can saturate camera sensors, creating false signals. This problem would be worse in summer, when satellites are visible for longer - introducing a seasonal bias that would harm LSST studies for which statistical significance must be built up over time, including studies of dark matter.

These issues can be managed, says Paul Dabbar, the under-secretary for science at the US Department of Energy, which funds the LSST camera. Operators could provide astronomers with detailed information on where the satellites are in the sky at any time, so that observers could schedule around expected satellite trails. Companies could also paint the crafts' Earth-facing surfaces a dull black, which would make them fainter.

SpaceX says that it is "taking steps to make the base of Starlink satellites black to help mitigate impacts on the astronomy community", but did not say whether this applies to the set just launched. The company also told Nature that it is sharing satellite-position information with the US military catalogue, and talking to astronomy groups to assess the effects and evaluate mitigation strategies.

\section{Radio interference}

Radio astronomers face a second set of challenges. They observe the Universe in

\section{“What's really concerning is how bright all these new satellite constellations will be."}

wavelengths of light that are also used for satellite communications. The use of such frequencies is regulated, but the huge number of planned satellites complicates the situation, says Tony Beasley, director of the US National Radio Astronomy Observatory in Charlottesville, Virginia. As satellites communicate with ground stations, their signals could interfere with radio-astronomy observations, rendering the astronomy data useless.
The observatory is talking to SpaceX and OneWeb about the frequencies that their megaconstellations will use for broadcasting. Companies might decide to shift these away from those used for radio astronomy. Another idea is for satellites to pause communications when they pass over radio-astronomy facilities.

The sheer number of satellites could also worsen Earth's space-junk problem. The first batch of Starlinks has already caused some congestion. In September, the European Space Agency (ESA) had to manoeuvre its Aeolus wind-mapping satellite out of the way of a Starlink craft. The Starlinks are supposed to move away from potential collisions automatically, but a communications glitch between ESA and SpaceX meant neither knew what the other was doing. The incident highlighted the fact that satellite operators don't have a universal strategy if two active satellites are on a potential collision course, says Holger Krag, head of ESA's space-debris office in Darmstadt, Germany.

He and his colleagues are hoping to help develop a global collision-avoidance system that automatically detects potential crashes and orders satellites to move to safer locations. "We would like to see that in two to three years," Krag says.

\title{
PROTON-SIZEPUZZLE LEAPSCLOSER TORESOLUTION
}

\section{Precise measurement affirms that the particle's radius is smaller than physicists once thought.}

\section{By Davide Castelvecchi}

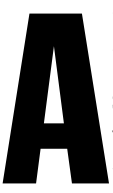
long-awaited experimental result has found the proton to be about $5 \%$ smaller than the previously accepted value. The finding ${ }^{1}$ has helped to prompt a redefinition of the particle's official size and seems to spell the end of the 'proton radius puzzle', which has enthralled physicists since 2010.

The result, published in Nature on 6 November, puts the particle's radius at 0.831 femtometres. This measurement, together with a concurring one made using a different technique that was published ${ }^{2}$ in Science in September, has been known to experts since last year. The findings led the Committee on Data for Science and Technology (CODATA) - which records the most up-to-date measurements of the fundamental constants of nature - to revise its handbook at the end of 2018, says Krzysztof Pachucki, a theoretical physicist at the University of Warsaw who chairs a CODATA task group. Although some researchers are still cautious, he thinks the latest papers have "definitely resolved the puzzle".

Physicists use two main techniques to measure the size of the proton. One relies on how electrons orbit atomic nuclei. Because some electron orbits pass through the protons in the nucleus, the size of the protons affects how strongly the electrons bind to the nucleus. Precise measurements of the differences between various electrons' energy levels - a technique known as spectroscopy - therefore provide a way to estimate the proton's radius. The second technique involves hitting atoms with a particle beam and seeing how those particles scatter off the nuclei.

About ten years ago, it seemed that both techniques had converged on a proton radius of 0.8768 femtometres (millionths of a millionth of a millimetre).

But in 2010, a new twist on spectroscopy 


\section{News in focus}

cast uncertainty on this consensus. At the Paul Scherrer Institute (PSI) in Villigen, Switzerland, physicists created exotic hydrogen atoms by replacing the electrons with muons, an elementary particle that is similar to electrons but 200 times more massive. Because muons spend more time inside the proton, their energy levels are affected more strongly than are those of the electrons. That means muon measurements of the proton's radius should be millions of times more precise than those made using ordinary hydrogen. The team measured a radius of 0.84184 femtometres ${ }^{3}$.

Randolf Pohl, who led that measurement and is now at the Johannes Gutenberg University in Mainz, Germany, has collaborated on other muonic experiments that have confirmed this value. For a while, researchers hoped that the discrepancy might reveal an unknown difference in how electrons and muons behave - something that could have upset the established quantum theory of electromagnetic phenomena.

More recently, however, improved spectroscopy experiments using ordinary hydrogen found a shrunken proton, suggesting that muons were not so special after all. Those efforts culminated in the Science paper ${ }^{2}$. After spending eight years perfecting a spectroscopy technique, the team behind that work found a radius of 0.833 femtometres - which is consistent with the value from the muon experiments.

\section{Converging results}

But more-conventional spectroscopy experiments done at Sorbonne University in Paris continued to disagree with this result ${ }^{4}$. And no one could explain why the scattering tech-

\section{“I don"t think the puzzle is quite solved yet, but we made some big advances."}

nique had pointed to a larger proton. Now, for the first time, a scattering experiment has found a smaller proton, too.

The latest experiment, called PRad, used an accelerator at the Thomas Jefferson National Accelerator Facility in Newport News, Virginia. PRad shoots a beam of electrons at hydrogen molecules, and measures how some of the electrons are deflected. Previous scattering experiments had used higher-energy electron beams, which have limited sensitivity to the proton radius, and then extrapolated to lower electron energies to determine the radius. That meant they had to make theoretical assumptions that might have skewed the final results. But the lower energies used by PRad circumvent the problem.

To further improve precision, PRad injected its hydrogen molecules directly into the vacuum pipe that carries the electron beam, rather than keeping it in a metal container as many previous experiments had done. This means there are no electrons hitting metal and confounding the measurement. Moreover, the team simultaneously measured how the beam scattered off not only the hydrogen's protons but also its electrons. Comparing the two types of scattering cancelled out another major source of error - fluctuations in hydrogen's density.

Ashot Gasparian, a particle and nuclear physicist at North Carolina A\&T State University in Greensboro who is the spokesperson for PRad, thinks he can still upgrade his experiment to further improve its precision.

But Jan Bernauer, a physicist at Stony Brook University in New York who has led earlier scattering measurements that found a larger proton, is not entirely convinced. “I don't think the puzzle is quite solved yet, but we made some big advances." He says that experiments in the pipeline, including one starting at PSI, will probably solve the puzzle once and for all.

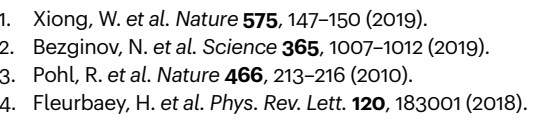

\section{nature masterclasses}

\section{Online Course in Scientific Writing and Publishing}

Delivered by Nature Research journal editors, researchers gain an unparalleled insight into how to publish.

Try a free sample of the course at masterclasses.nature.com

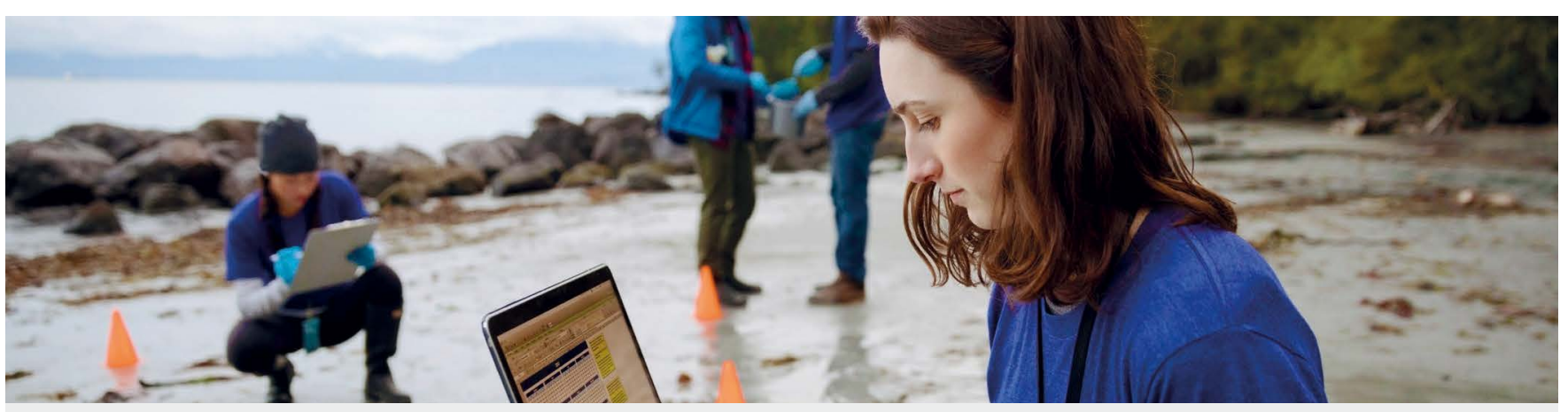

Bite-size design for busy researchers • Subscribe as a lab or institution 